\title{
Size-dependent single-particle energy levels and interparticle Coulomb interactions in CdSe quantum dots measured by scanning tunneling spectroscopy
}

\author{
Lucian Jdira, ${ }^{1}$ Peter Liljeroth, ${ }^{2}$ Eric Stoffels, ${ }^{1}$ Daniël Vanmaekelbergh, ${ }^{2}$ and Sylvia Speller ${ }^{1}$ \\ ${ }^{1}$ Institute for Molecules and Materials, Radboud University Nijmegen, P.O. Box 9010, 6500 GL Nijmegen, The Netherlands \\ ${ }^{2}$ Condensed Matter and Interfaces, Debye Institute, University of Utrecht, P.O. Box 80000, 3508 TA Utrecht, The Netherlands
}

(Received 11 November 2005; revised manuscript received 17 January 2006; published 3 March 2006)

\begin{abstract}
We report on tunneling spectroscopy measurements on colloidal CdSe quantum dots of different sizes. The size-dependent energy level structure and electron-hole Coulomb attraction in CdSe quantum dots are obtained by a combination of shell-tunneling spectroscopy and optical spectroscopy. The results are in good agreement with tight-binding calculations. The electron-electron interactions are investigated by shell-filling spectroscopy. The tunneling spectra in this regime are analyzed by solving the master equation for the electron and hole occupancy of the quantum dot.
\end{abstract}

DOI: 10.1103/PhysRevB.73.115305

PACS number(s): 73.22.-f, 73.21.La, 73.63.Kv, 68.37.Ef

\section{INTRODUCTION}

Semiconductor quantum dots (QDs) provide the opportunity to study the evolution of electronic properties on the length scale intermediate between the molecular and the solid-state regimes. ${ }^{1}$ Due to quantum confinement, the QDs, sometimes called "artificial atoms," have discrete electronic levels that shift to higher energies with decreasing size. ${ }^{2}$ Optical absorbance, luminescence, and excitation spectroscopies have been used to study in detail the dependence of the optical transitions on the nanocrystals size, shape, and material. ${ }^{3-7}$

It is, however, difficult to separately investigate electron and hole energy levels by optical spectroscopy as it inherently involves transitions between two levels, and not individual states. Resonant tunneling spectroscopy can overcome this limitation, and recently scanning tunneling spectroscopy has been used to probe the energy levels of semiconductor nanocrystals (InAs, ${ }^{8-11} \mathrm{CdSe},{ }^{12,13}$ and $\mathrm{PbSe}^{14}$ ). Tunneling spectroscopy is able to provide unique information on the electronic properties of the nanocrystals as it can probe the complete density of states in the absence of selection rules. ${ }^{5,14}$

An important parameter in tunneling spectroscopy is the number of additional electrons (holes) in the nanocrystal. In the limiting case, called shell-tunneling spectroscopy, where the rate of tunneling into the nanostucture, $\Gamma_{\text {in }}$, is much smaller than the rate of tunneling out, $\Gamma_{\text {out }}$, electrons (holes) tunnel through the QD one-by-one and interparticle interactions do not occur. ${ }^{5,12}$ In this case, the measured spectrum directly reflects the single-particle density of states of the nanocrystal. When $\Gamma_{\text {in }}$ is increased and becomes comparable to $\Gamma_{\text {out }}$, the degeneracy of the levels is lifted due to interactions between the carriers accumulating in the dot. This results in additional peaks in the tunneling spectrum and makes it possible to obtain information on the interparticle Coulomb interaction energies. ${ }^{8,12}$ This case is called (partial) shell-filling spectroscopy.

Scanning tunneling spectroscopy has been previously performed on colloidal CdSe nanocrystals; however, the studies have only focused on a single QD size. ${ }^{12,15,16}$ In a previous paper, ${ }^{12}$ we reported the energetic structure of $4.3 \mathrm{~nm} \mathrm{CdSe}$ QD as observed by scanning tunneling spectroscopy. Here, we present a detailed analysis of the spectra for CdSe QDs with diameters between 2.6 and $5 \mathrm{~nm}$. We vary the width of the tip/dot tunneling barrier and show that relative rates of tip-to-dot and dot-to-substrate tunneling critically determine the electron occupation in the CdSe dots. When the measurement is carried out with the tip sufficiently retracted from the dot, the electrons tunnel one at a time through the QD and the peaks in the conductance spectrum correspond to the single-particle levels. ${ }^{12,14}$ The measured single-particle energy levels agree well with values predicted by tight-binding calculations. Combining the results from scanning tunneling spectroscopy and optical spectroscopy yields the electronhole Coulomb energy. Finally, we show how the tunneling spectrum is changed when the tip is brought closer to the dot and Coulomb interactions between electrons occur. In this case, detailed understanding of the tunneling spectra requires taking into account simultaneous tunneling of both electrons and holes.

\section{TUNNELING SPECTRA ON SINGLE, ISOLATED CdSe QUANTUM DOTS}

CdSe nanocrystals were prepared according to the literature methods. ${ }^{17}$ These dots are nearly spherical and have a size dispersion of $5-10 \%$. The surface of the nanocrystals is passivated by organic ligands; the ligand shell of the assynthesized nanocrystals (tetraoctylphosphine oxide and hexadecylamine) was exchanged to pyridine (3 and $5 \mathrm{~nm}$ diameter nanocrystals) or hexylamine $(2.6 \mathrm{~nm}$ diameter nanocrystals). ${ }^{18} \mathrm{~A}$ flame-annealed $\mathrm{Au}(111)$ substrate with a self-assembled hexanedithiol monolayer ${ }^{19}$ was immersed in a dispersion of colloidal CdSe QDs for $1 \mathrm{~min}$. This leads to a stable attachment of isolated dots and the formation of small aggregates of a few dots. The density of the nanocrystals on the surface can be controlled by the QDs concentration in the solution and/or the immersion time. We have investigated about 10-20 isolated QDs of each size (on about seven different samples). The diameter of the dots, between 2.6 and 


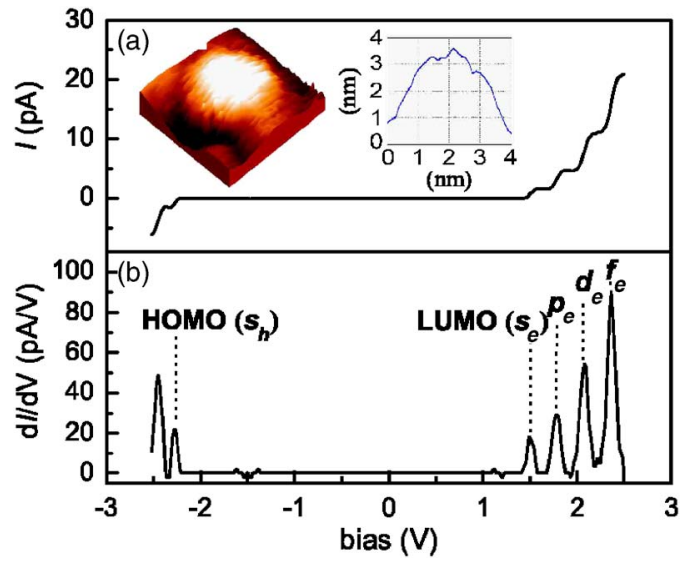

FIG. 1. (Color online) (a) Example of a single $I-V$ curve measured on an isolated CdSe QD: $4.5 \mathrm{~K}$, tip-dot distance stabilized at $20 \mathrm{pA}$ and $2.5 \mathrm{~V}$. The inset shows the image of an isolated QD recorded at $V_{\text {bias }}=2.5 \mathrm{~V}$ and $I_{\text {set-point }}=5 \mathrm{pA}$. (b) The tunneling spectrum obtained by digitally filtering and differentiating the experimental $I-V$ curve.

$5 \mathrm{~nm}$, was estimated from the optical gap measured in the dispersion using literature data. ${ }^{20}$

The scanning tunneling microscopy (STM) work was performed in a home-built cryogenic STM at $4.5 \mathrm{~K}$ with cut $\mathrm{Pt} / \mathrm{Ir}$ tips. The scanning head and sample are evacuated before introducing helium exchange gas and inserting the STM in the liquid helium bath. The STM image of an isolated $3 \mathrm{~nm}$ diameter CdSe QD recorded at a sample voltage $V_{\text {bias }}$ $=2.5 \mathrm{~V}$ and tunneling current $I_{\text {set-point }}=5 \mathrm{pA}$ is shown in the inset of Fig. 1(a). The dot is well separated from its neighbors and it has a well-defined spherical shape. The mean value of the apparent height and the full width at half maximum were found to be 2.5 and $3.0 \mathrm{~nm}$, respectively. Al- though STM topography provides only an estimate of the size, we can conclude that the shape and height are compatible with the size expected from optical measurements of the colloidal solution of the nanocrystals.

Tunneling spectra were acquired at $4.5 \mathrm{~K}$ by placing the STM tip above the center of an isolated dot well-separated from the neighboring nanocrystals and disconnecting the feedback loop. The tunneling current $I$ is measured as a function of the tip-substrate potential difference (bias) $V$. The tip-QD junction is controlled via the STM feedback settings while the barrier between QD and $\mathrm{Au}(111)$ substrate is fixed and is due to the hexanedithiol layer. Typically, a large number (100 for each set-point current) of curves were acquired above a single dot and checked for their reproducibility. The $I-V$ curves were reproducible, although discrete shifts of the curves along the voltage axis were observed in some cases. The reason for this could be the trapping of a charge in the surroundings of the dot that acts as a local "gate" and leads to a shift of the measured spectrum. Since current flow results from resonant tunneling via the QD orbitals, a plot of the differential conductance $d I / d V$ as a function of $V$ (tunneling spectrum) shows a sequence of peaks, reflecting the electron and hole energy levels in the quantum dot. Figure 1(a) shows a typical single $I-V$ curve acquired on a $\mathrm{CdSe}$ QD, $3 \mathrm{~nm}$ in diameter, exhibiting steplike behavior. The tunneling spectrum, Fig. 1(b), was obtained by digitally filtering and differentiating the experimental $I-V$ curve. The $d I / d V$ curves show a very high signal-to-noise ratio, indicating the high quality of our spectra. This allows us to measure directly in the integrated mode without lock-in amplification.

In Fig. 2, typical tunneling spectra of CdSe dots of three different nominal diameters are shown: (a) $5 \mathrm{~nm}$, (b) $3 \mathrm{~nm}$, and (c) $2.6 \mathrm{~nm}$. We varied the tunneling resistance between the tip and the nanocrystal by changing the set-point current between 10 and $100 \mathrm{pA}$ at a bias of $2.5 \mathrm{~V}$. As can be seen in

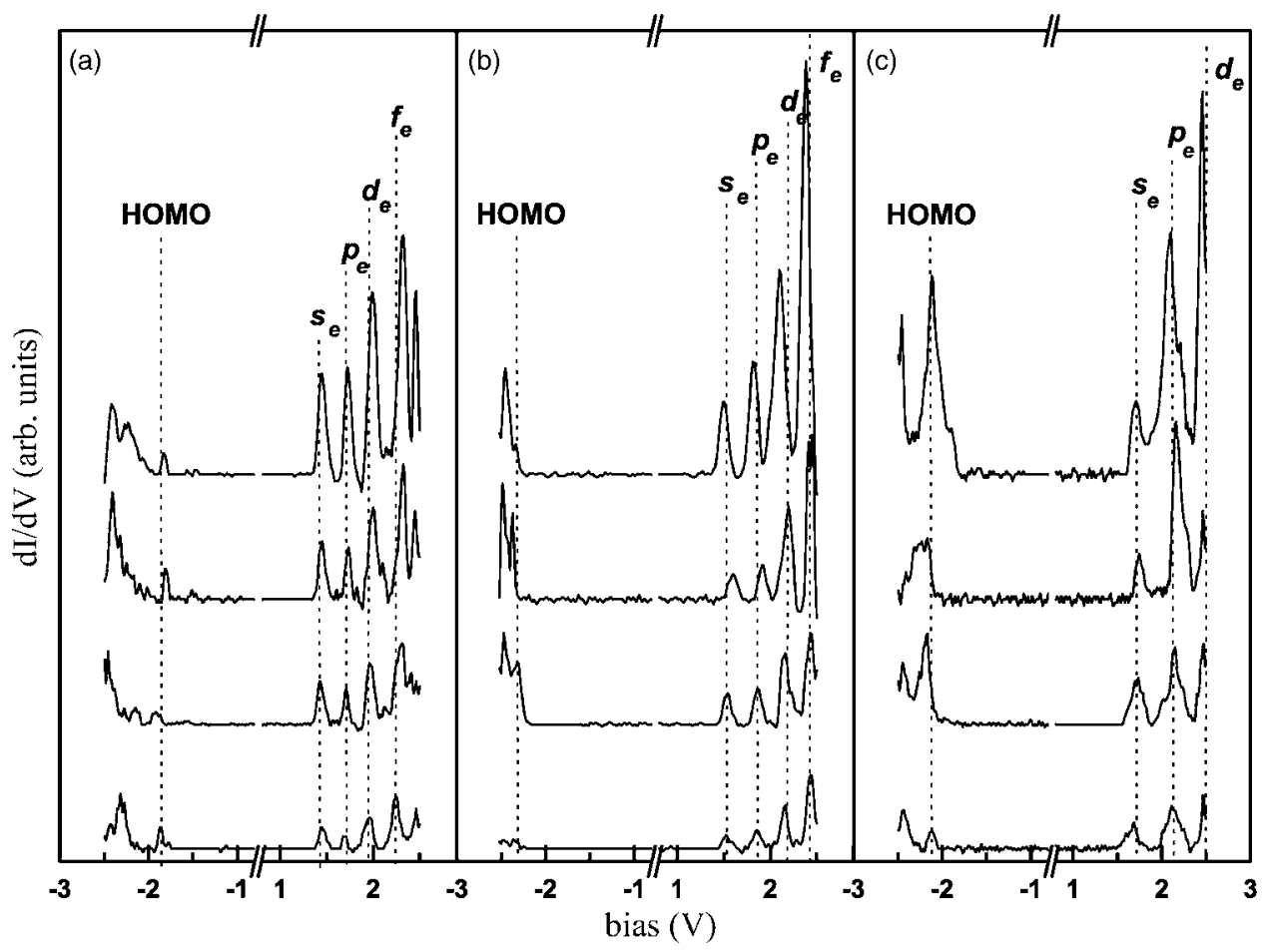

FIG. 2. Size evolution of the tunneling spectra $d I / d V$ vs $V$ characteristics of isolated $\mathrm{CdSe}$ QDs in the shell-tunneling range: $4.5 \mathrm{~K}$, (a) $5 \mathrm{~nm}$ diameter nanocrystal, set-point 10, 20,30, and $60 \mathrm{pA}$; (b) $3 \mathrm{~nm}$, set-point 10, 20, 70 , and $100 \mathrm{pA}$; (c) $2.6 \mathrm{~nm}$, setpoint $10,20,30$, and $80 \mathrm{pA}$-at $2.5 \mathrm{~V}$. The peaks in the positive voltage range correspond to tunneling through $s_{e}$ (LUMO), $p_{e}, d_{e}$, and $f_{e}$ electron orbitals. The peaks in the negative bias range correspond to tunneling through the HOMO and other hole states. The peaks do not shift and the peak intensity is increased with increasing the set-point current (decreasing the tip-dot distance). 
Fig. 2, this did not affect the peak position in the measured spectra; only the peak intensity is increased by decreasing tip-QD separation (increasing set-point current). Several conductance peaks can be seen in the negative bias range, corresponding to the first valence orbitals (hole levels) of the CdSe QDs. In the positive bias range, the peaks correspond to the conduction orbitals (electron levels) and increase in intensity with increasing energy. Both the zero-conductivity gap [difference between the highest occupied molecular orbital (HOMO) and the lowest unoccupied molecular orbital (LUMO)] as well as the level separations increase with decreasing dot diameter. The last resonances at positive bias cannot be detected with the smallest QDs as the tunneling conditions become unstable at biases exceeding $2.5 \mathrm{~V}$. We infer that the spectra shown in Fig. 2 are shell-tunneling spectra; this conclusion will be justified in the next section.

\section{SINGLE-ELECTRON ENERGY LEVELS FROM SHELL-TUNNELING SPECTRA}

The size range studied in this paper corresponds to the strong confinement regime, where the confinement energy has a significant influence on the spectral features. The resonant tunneling response of the system is determined by the level structure and electron-electron interactions.

At the first resonance at positive bias, the probability to find one electron in the LUMO of the QD is

$$
P\left(s^{1}\right)=\frac{2 \Gamma_{s}^{\text {in }}}{\left(2 \Gamma_{s}^{\text {in }}+\Gamma_{s}^{\text {out }}\right)},
$$

where $\Gamma_{s}^{\text {in }}$ is the rate at which an electron tunnels from the tip into the empty $s_{e}$ orbital, and $\Gamma_{s}^{\text {out }}$ is the tunneling rate from the occupied $s_{e}$ orbital into the substrate electrode.

In the shell-tunneling case $\Gamma_{s}^{\text {in }} \ll \Gamma_{s}^{\text {out }}$; therefore, $P\left(s^{1}\right) \cong 0$, and one electron tunnels through the dot at a time. The tunneling current that increases with decreasing tip-dot distance is then

$$
I=n_{e} e \Gamma_{s}^{\mathrm{in}},
$$

where $n_{e}$ is the number of available electron channels. Tunneling leads to polarization of the dot by a single electron (polarization energy), but Coulomb interactions between two (or more) additional electrons do not occur. This still holds when the electrochemical potential of the tip electrode is increased further, such that tunneling from the tip to the higher levels (e.g., $p$-type level) occurs.

In order to obtain quantitative information from the tunneling spectra, the distribution of the bias voltage over the double-barrier tunnel junction has to be known. We calculated this by solving the Poisson equation for a realistic tipdot-substrate geometry as shown in Fig. 3(a), with the dielectric constant 8 for $\mathrm{CdSe}$ and 2.1 for organic molecules. We found that $\eta=\left(V_{\text {dot }}-V_{\text {tip }}\right) / V_{\text {bias }}$ is on average 0.74 for dot sizes between 2 and $5 \mathrm{~nm}$ and realistic tip-to-dot and dot-tosubstrate distances in the shell-tunneling regime. This implies that most of the potential drops between the tip and the dot. Then, the Fermi level of the tip is successively in resonance with the various electronic levels of the QD and the (a)
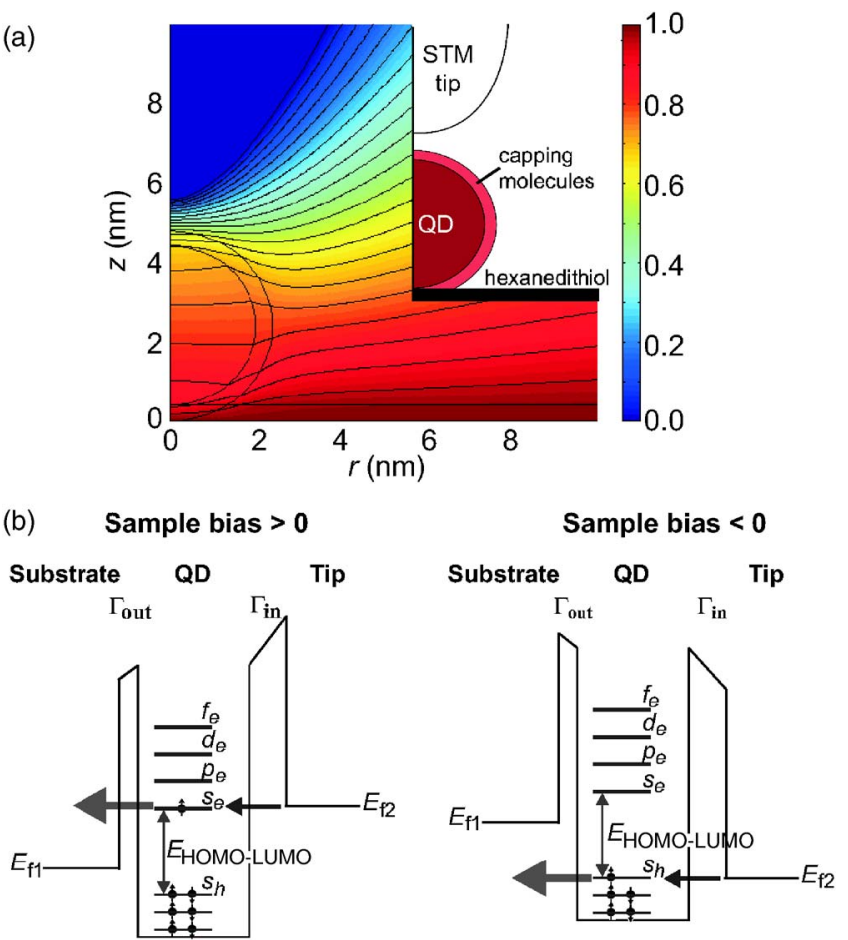

FIG. 3. (Color online) (a) Potential distribution in the tip-QDsubstrate double barrier tunnel junction calculated by the solution of the Poisson equation in a realistic geometry. (b) Schemes representing the principles of tunneling of electrons through the conduction levels at positive bias (left) and tunneling of holes through the valence levels at negative bias (right) in the shell-tunneling regime.

peaks in the measured spectrum directly reflect the energy level positions, Fig. 3(b). The "conduction band" states appear at positive and "valence band" states at negative bias, respectively. Moreover, changing of the set point varies all the peak amplitudes by the same factor, the peak positions remain unchanged, and no new peaks appear in the spectra. This is exactly what we observe at low set points, as can be seen from Fig. 2. Hence, the peaks at positive bias correspond to tunneling through the electron orbitals of the $\mathrm{CdSe}$ QD $s, p, d$, and $f$ symmetry in order of increasing energy. The small peak observed in some spectra between $d_{e}$ and $f_{e}$ levels should correspond to the second $s_{e}$ level (see Fig. 2, $5 \mathrm{~nm}$ QD). The peak amplitudes become larger with increasing energy, reflecting the increased transmission through orbitals with larger spatial extension and degeneracy. Table I summarizes the transitions between the ground state and the different one-electron excited states, corresponding to the peaks in the shell-tunneling spectra.

The bias voltage at resonance in the tunneling spectrum is related to the energy levels, $E_{i}$, through

$$
\eta V_{\text {bias }}=E_{i}+\Sigma_{i}
$$

where $\Sigma_{i}$ is the polarization energy of an electron on level $i$. This energy depends on the dielectric mismatch between the nanocrystal core and its surroundings and can be estimated given the dielectric constant of the nanocrystal, $\epsilon_{\text {in }}$, and the effective value for the environment, $\epsilon_{\text {out }}{ }^{21-24}$ 
TABLE I. State transitions in the CdSe QDs corresponding to the peaks in the shell-tunneling spectra (Fig. 2). The state transitions involve $s_{h}$ (HOMO), $s_{e}$ (LUMO), $p_{e}, d_{e}$, and $f_{e}$ orbitals. The third column gives the chemical potentials of the state transition: $E_{i}$ and $\Sigma_{i}$ stand for the energy and the polarization energy of an electron in orbital $i$.

\begin{tabular}{ccc}
\hline \hline Peak symbol & Transition type & Chemical potential \\
\hline $\operatorname{HOMO}\left(s_{h}\right)$ & $s_{h}^{0} \rightarrow s_{h}^{1}$ & $E_{s_{h}}-\Sigma_{s_{h}}$ \\
$\operatorname{LUMO}\left(s_{e}\right)$ & $s_{e}^{0} \rightarrow s_{e}^{1}$ & $E_{s_{e}}+\Sigma_{s_{e}}$ \\
$p_{e}$ & $s_{e}^{0} p_{e}^{0} \rightarrow s_{e}^{0} p_{e}^{1}$ & $E_{p_{e}}+\Sigma_{p_{e}}$ \\
$d_{e}$ & $s_{e}^{0} d_{e}^{0} \rightarrow s_{e}^{0} d_{e}^{1}$ & $E_{d_{e}}+\Sigma_{d_{e}}$ \\
$f_{e}$ & $s_{e}^{0} f_{e}^{0} \rightarrow s_{e}^{0} f_{e}^{1}$ & $E_{f_{e}}+\Sigma_{f_{e}}$ \\
\hline \hline
\end{tabular}

$$
\Sigma(R)=\frac{\epsilon_{\mathrm{in}}-\epsilon_{\mathrm{out}}}{\epsilon_{\mathrm{in}}\left(\epsilon_{\mathrm{in}}+\epsilon_{\mathrm{out}}\right)}(1 / \alpha-0.376 \alpha+0.933) \frac{e^{2}}{8 \pi \epsilon_{0} R},
$$

where $\alpha=\epsilon_{\text {out }} /\left(\epsilon_{\text {in }}+\epsilon_{\text {out }}\right)$. For nanocrystals used in this study, the polarization energy varied between $229 \mathrm{meV}$ (diameter $2.6 \mathrm{~nm}$ ) and $119 \mathrm{meV}$ (diameter $5 \mathrm{~nm}$ ) for $\epsilon_{\text {in }}=8$ and $\epsilon_{\text {out }}$ $=2.1$. The zero-conductance gap in the tunneling spectrum, $\Delta V_{\mathrm{ZC}}^{\mathrm{STS}}$ (also called the quasiparticle gap), and the optical gap, $E_{\text {gap }}^{\mathrm{opt}}$, are related to the HOMO-LUMO single particle gap, $\Delta E_{s_{e}-s_{h}}$, as follows:

$$
\eta \Delta V_{\mathrm{ZC}}^{\mathrm{STS}}=\Delta E_{s_{e^{-S} h}}+\Sigma_{s_{e}}+\Sigma_{s_{h}}=E_{\mathrm{gap}}^{\mathrm{opt}}+J_{e-h},
$$

where $J_{e-h}$ is electron-hole Coulomb attraction energy. We combined the STS and optical measurements and using the calculated values for polarization energy, the electron-hole Coulomb energy can be extracted. This varies from $0.20 \mathrm{eV}$ ( $5 \mathrm{~nm}$ diameter) to $0.73 \mathrm{eV}$ (2.6 nm diameter) for the measured dots. The $e-h$ attraction energy can be estimated in a similar manner to the polarization energy, ${ }^{21,24}$

$$
J_{e-h}(R)=\left(\frac{1}{\epsilon_{\mathrm{out}}}+\frac{0.79}{\epsilon_{\mathrm{in}}}\right) \frac{e^{2}}{4 \pi \epsilon_{0} R} .
$$

Figure 4 shows good agreement between the measured values (points) with theoretical predictions (line). This indicates

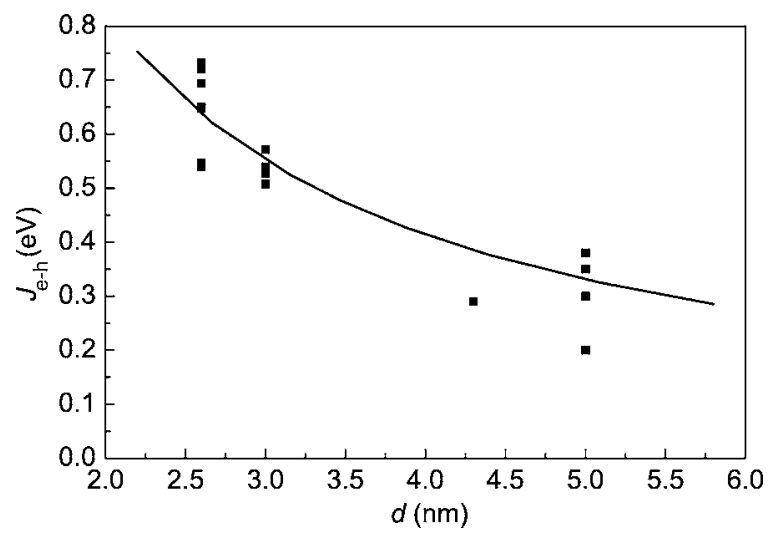

FIG. 4. The size dependence of $J_{e-h}$ Coulomb energies, our data, and the data from Ref. 12 (4.3 nm QD diameter). The line refers to electrostatic calculations (Ref. 21).

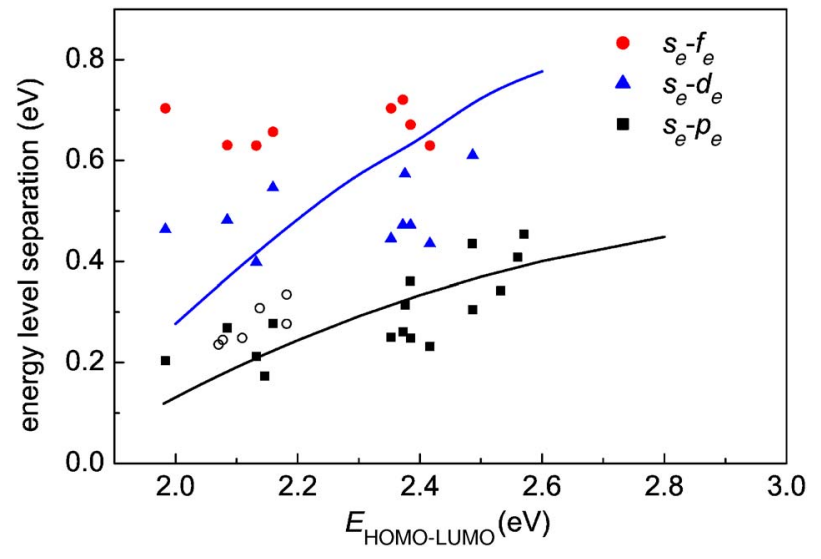

FIG. 5. (Color online) Energy levels separation as a function of HOMO-LUMO gap (filled symbols): $E_{p_{e}}-E_{s_{e}}$ (squares); $E_{d_{e}}-E_{s_{e}}$ (triangles); $E_{f_{e}}-E_{s_{e}}$ (circles). The solid lines are theoretical values for $s-p$ and $s-d$ separation energies by tight-binding calculations (Ref. 25) The open symbols are literature values for the $s-p$ level separation based on intraband adsorption measurements in charged quantum dot solids (Refs. 26-28).

that the calculated potential distribution across the tip/dot/ substrate double barrier tunnel junction is realistic and that the HOMO-LUMO gap measured by STS is consistent with the optical measurements. The $\epsilon_{\text {out }}$ that we assume is in good agreement with the value which we found for PbSe dots attached with the same organic linker to $\mathrm{Au}(111)$ substrate. ${ }^{14}$ The low value of $\epsilon_{\text {out }}$ indicates that screening due to the STM tip or the substrate is not significant in the shell-tunneling regime. We note that the Coulomb attraction energy is not the same as the exciton $(e, h)$ binding energy.

In Fig. 5, the energy levels separation derived from the resonant tunneling spectra measurements are shown: the $s_{e}$ $-p_{e}, s_{e}-d_{e}$, and $s_{e}-f_{e}$ level separation have been plotted as a function of the HOMO-LUMO single particle gap (filled symbols). The HOMO-LUMO single-particle gap was chosen as the $x$ axis because the energy is more easily and precisely measured than the dot size. The bias voltage has been converted to the energy scale using $\eta=0.74$, and polarization energies were calculated using $\epsilon_{\text {out }}=2.1$, see above. The experimental data are compared with the energy levels separation calculated by tight-binding theory ${ }^{25}$ (solid lines) and literature values for the $s_{e}-p_{e}$ level separation in CdSe QDs based on intraband adsorption measurements in charged quantum dot solids ${ }^{26-28}$ (open symbols). There is fair agreement between theory and experiment. Possible reasons for the scattering in the measured values find their origin in a number of experimental uncertainties. The first is related to the size distribution of the CdSe QDs $( \pm 5 \%)$ leading to $\pm 10 \%$ uncertainties in the energy difference between energy levels. The second uncertainty is related to the shape: it is not possible to detect small deviations from a spherical shape with STM.

\section{INTERPRETATION OF (PARTIAL) SHELL-FILLING SPECTRA}

Figure 6(a) shows a typical spectrum of a $3 \mathrm{~nm}$ CdSe QD with the tip-dot distance stabilized at $240 \mathrm{pA}$ and $2.5 \mathrm{~V}$. In 


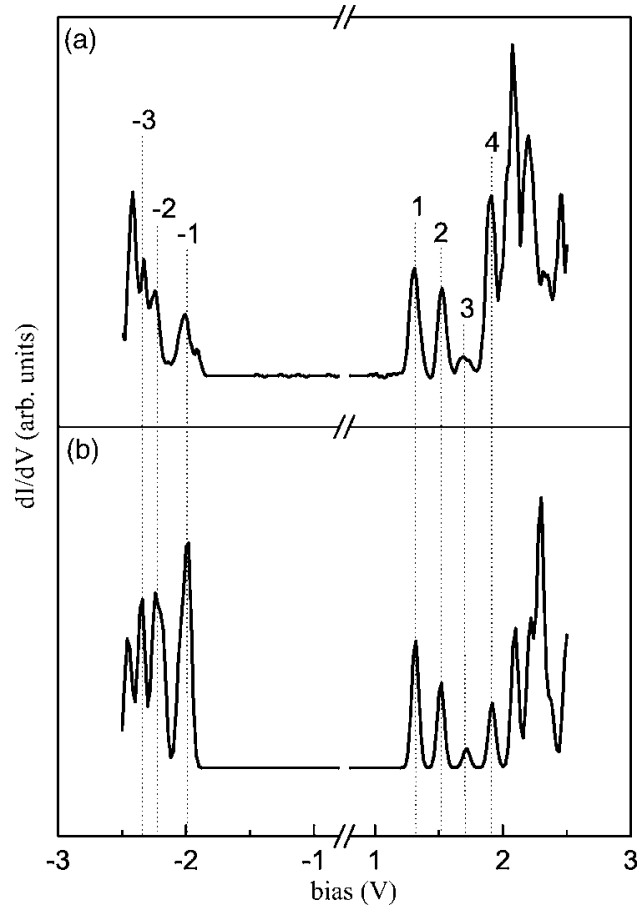

FIG. 6. (a) Typical spectra for $3 \mathrm{~nm}$ CdSe QD measured with the tip-dot distance stabilized closer to the dot (set-point current $240 \mathrm{pA}$ at $2.5 \mathrm{~V}$ ). The spectra show more peaks, indicating lifting of the orbital degeneracy. (b) Theoretical simulation of the tunnelling spectra with (partial) shell-filling conditions.

the positive bias range, we first find three closely spaced peaks, decreasing in intensity, followed by a group of peaks with higher intensity. The fact that we observe more resonances than at low set-point currents points to the breaking of the spin and orbital degeneracy due to electronelectron Coulomb interactions in the QD. In other words, more than one electron is present in the dot at a given time. In the negative bias range, we first find a peak at about $-1.9 \mathrm{~V}(-1)$, followed by a group of peaks. In general, the resonances at negative bias reflect tunneling of holes through valence levels. However, some peaks at negative bias may arise from tunneling of electrons via the conduction levels. ${ }^{21}$ In addition to experiments with colloidal nanocrystals, this type of bipolar transport has been recently observed with STM in a molecular system of copper phthalocyanine adsorbed on an ultrathin oxide layer on $\mathrm{NiAl}(110)$ surface. ${ }^{21,29}$ Bipolar transport is expected if, due to the voltage distribution in the tunneling gap, the QD levels are shifted up sufficiently to bring the first conduction level in resonance with the substrate Fermi level before the tip Fermi level reaches resonance with the first valence level (see Fig. 7). In the case of concomitant electron and hole tunneling (electrons tunneling on both sides of the zero-conductance gap), $\eta$ can be estimated from the positions of the first peaks on the negative and positive bias. ${ }^{21}$ This gives $\eta=0.61$ for the spectrum in Fig. 6(a).

In order to unravel the complex response in the partial shell-filling regime, we have simulated the tunneling spectra taking into account simultaneous tunneling of holes and electrons using the master equation approach. ${ }^{21}$ The input param-
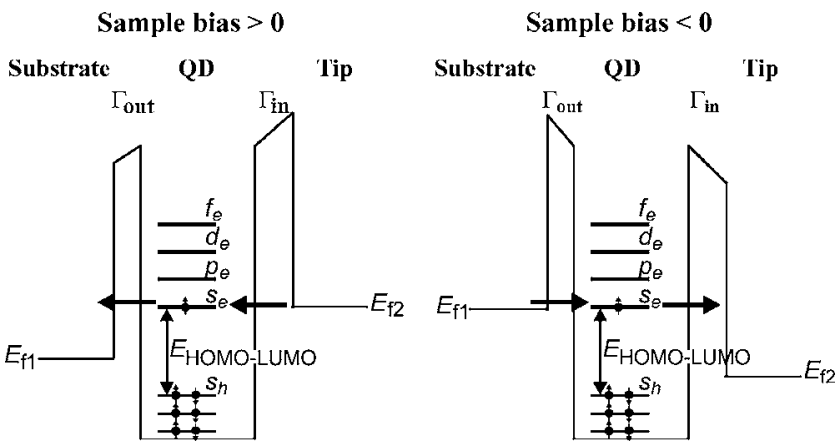

FIG. 7. Schemes representing the principles of tunneling at positive (left) and negative (right) bias through both conduction levels and valence levels, respectively, of the QD (partial shell-filling regime).

eters for the simulation are the level positions, $\eta$ (taken to be 0.61 in the shell-filling regime), polarization and charging energies, and the tunneling rates in and out of the QD. The simulated response is shown in Fig. 6(b). The spectrum was calculated using $T=0 \mathrm{~K}$ and convoluted by a $25 \mathrm{meV}$ Gaussian. The tunneling rates used were $\Gamma_{\text {in }}=2 \times 10^{8} 1 / \mathrm{s}$ and $\Gamma_{\text {out }}=1 \times 10^{8} 1 / \mathrm{s}$ for the electrons and $\Gamma_{\text {in }}=0.5 \times 10^{8} 1 / \mathrm{s}$ and $\Gamma_{\text {out }}=0.25 \times 10^{8} 1 / \mathrm{s}$ for the holes. These rates correspond to very weak coupling between the dot and the substrate or the tip. The first two peaks on the positive bias are easily interpreted as tunneling through the $s_{e}$ level, which is split by the electron-electron Coulomb interaction. The third peak corresponds to an excited state, tunneling through the $p_{e}$ level with empty $s_{e}$ level. By comparing the theoretical predictions while varying the tunneling rates (between complete shell-tunneling and shell-filling), we are able to assign the peak 4 to tunneling through the $p_{e}$ state with one electron already present in the QD. The group of peaks after this is a combination of tunneling through $p_{e}$ levels with several added electrons in the QD and tunneling through the hole levels. The details of the simulations and bipolar transport in nanocrystal systems will be reported in a future publication.

On negative bias, we find that the first peak indeed corresponds to tunneling through the first conduction level (Fig. 7, right), followed by a mixture of tunneling through conduction and valence levels. Table II shows the assignment of the peaks in terms of specific transitions between two states of the QD. The third column gives the chemical potential for the transitions. It follows from Table II that the energy difference between peaks 1 and 2 is equal to the Coulomb energy between the two electrons in the $s_{e}$ orbital, $J_{s-s}$. The energy difference between peaks 3 and 1 is nearly equal to $E_{p_{e}}-E_{s_{e}}$, since $\left(E_{p_{e}}-E_{s_{e}}\right) \gg\left(\Sigma_{p_{e}}-\Sigma_{s_{e}}\right)$. For a value of $\eta$ $=0.61$, we find $J_{s-s}=0.137 \mathrm{eV}$ and $E_{p_{e}}-E_{s_{e}}=0.236 \mathrm{eV}$; the $s_{e}-p_{e}$ level separation is in agreement with the value obtained from shell-tunneling spectra. The energy difference between peaks 4 and 3 is equal to the Coulomb energy between one electron in the $s$ orbital and one in the $p$ orbital, $J_{s-p}=0.122 \mathrm{eV}$. In principle, the electron-electron repulsion energy should be very close to the electron-hole attraction energy, which is $\sim 0.5 \mathrm{eV}$ for this size (compare with Fig. 4, $3 \mathrm{~nm}$ dot). However, in accordance with an earlier study, we find that the $J_{e-e}$ measured by shell-filling spectroscopy is 
TABLE II. State transitions in the CdSe QD corresponding to the peaks in the partial shell-filling spectra (Fig. 6) involving electron levels $\left(s_{e}, p_{e}\right)$. The third column gives the chemical potential of the state transition (neglecting exchange interaction): $E_{i}$ stands for the energy of the confinement level $i, \Sigma_{i}$ for the polarization energy of an electron or hole in orbital $i$, and $J_{i-j}$ for the Coulomb repulsion energy between electrons on levels $i$ and $j$.

\begin{tabular}{ccc}
\hline \hline Peak symbol & Transition & Chemical potential \\
\hline-1 & $s_{e}^{0} \rightarrow s_{e}^{1}$ & \\
1 & $s_{e}^{0} p_{e}^{0} \rightarrow s_{e}^{1} p_{e}^{0}$ & $E_{s_{e}}+\Sigma_{s_{e}}$ \\
2 & $s_{e}^{1} p_{e}^{0} \rightarrow s_{e}^{2} p_{e}^{0}$ & $E_{s_{e}}+\Sigma_{s_{e}}+J_{s-s}$ \\
3 & $s_{e}^{0} p_{e}^{0} \rightarrow s_{e}^{0} p_{e}^{1}$ & $E_{p_{e}}+\Sigma_{p_{e}}$ \\
4 & $s_{e}^{1} p_{e}^{0} \rightarrow s_{e}^{1} p_{e}^{1}$ & $E_{p_{e}}+\Sigma_{p_{e}}+J_{s-p}$ \\
\hline \hline
\end{tabular}

significantly smaller than $J_{e-h}$ measured by shell-tunneling spectroscopy. ${ }^{12}$ A possible reason for this discrepancy is screening due to the STM tip that is brought closer to the QD in order to reach the shell-filling regime.

\section{CONCLUSIONS}

We have investigated in detail the size dependence of the quantum confined levels in colloidal CdSe nanocrystals us- ing scanning tunneling spectroscopy. In the shell-tunneling regime, the complete single-particle density of states can be measured in the absence of selection rules. The measured level separations are in good agreement with tight-binding calculations. When the tunneling rate into the $\mathrm{QD}$ is increased, the interparticle interactions can be probed with shell-filling spectroscopy. The observed transitions in the tunneling spectra can be explained by a master equation accounting for the electron-hole occupation of the discrete conduction and valence orbitals of the CdSe QD.

\section{ACKNOWLEDGMENTS}

This work is supported by the Netherlands Foundation for Fundamental Research on Matter (FOM), by NanoNed, the Dutch nanotechnology programme of the Ministry of Economic Affairs, and by the European projects "Advanced Scanning Probes for Innovative Nanoscience and Technology" (ASPRINT, NMP-CT-2003-001601) and "Tunneling spectroscopy on single molecular and semiconducting nanostructures" (NANOSPECTRA, HPRH-CT-2001-00320). We gratefully acknowledge Arjan Houtepen and Celso de Mello Donegá for the nanocrystal samples used in this study and Jan G.H. Hermsen for technical support with the experimental setup.
${ }^{1}$ A. P. Alivisatos, Science 271, 933 (1996).

${ }^{2}$ L. E. Brus, J. Chem. Phys. 80, 4403 (1984).

${ }^{3}$ A. L. Efros and M. Rosen, Annu. Rev. Mater. Sci. 30, 475 (2000).

${ }^{4}$ C. B. Murray, C. R. Kagan, and M. G. Bawendi, Annu. Rev. Mater. Sci. 30, 545 (2000).

${ }^{5}$ U. Banin and O. Millo, Annu. Rev. Phys. Chem. 54, 465 (2003).

${ }^{6}$ M. A. El-Sayed, Acc. Chem. Res. 37, 326 (2004).

${ }^{7}$ C. Burda, X. Chen, R. Narayanan, and M. A. El-Sayed, Chem. Rev. (Washington, D.C.) 105, 1025 (2005).

${ }^{8}$ U. Banin, Y. Cao, D. Katz, and O. Millo, Nature (London) 400, 542 (1999).

${ }^{9}$ O. Millo, D. Katz, Y. W. Cao, and U. Banin, Phys. Rev. Lett. 86, 5751 (2001).

${ }^{10}$ B. Grandidier, Y. M. Niquet, B. Legrand, J. P. Nys, C. Priester, D. Stievenard, J. M. Gerard, and V. Thierry-Mieg, Phys. Rev. Lett. 85, 1068 (2000).

${ }^{11}$ T. Maltezopoulos, A. Bolz, C. Meyer, C. Heyn, W. Hansen, M. Morgenstern, and R. Wiesendanger, Phys. Rev. Lett. 91, 196804 (2003).

${ }^{12}$ E. P. A. M. Bakkers, Z. Hens, A. Zunger, A. Franceschetti, L. P. Kouwenhoven, L. Gurevich, and D. Vanmaekelbergh, Nano Lett. 1, 551 (2001).

${ }^{13}$ D. Katz, T. Wizansky, O. Millo, E. Rothenberg, T. Mokari, and U. Banin, Phys. Rev. Lett. 89, 086801 (2002).

${ }^{14}$ P. Liljeroth, P. A. Zeijlmans van Emmichoven, S. G. Hickey, H. Weller, B. Grandidier, G. Allan, and D. Vanmaekelbergh, Phys. Rev. Lett. 95, 086801 (2005).

${ }^{15}$ E. P. A. M. Bakkers and D. Vanmaekelbergh, Phys. Rev. B 62,
R7743 (2000).

${ }^{16}$ R. Bernard, G. Comtet, G. Dujardin, V. Huc, and A. J. Mayne, Appl. Phys. Lett. 87, 053114 (2005).

${ }^{17}$ C. de Mello Donegá, S. G. Hickey, S. F. Wuister, D. Vanmaekelbergh, and A. Meijerink, J. Phys. Chem. B 107, 489 (2003).

${ }^{18}$ C. B. Murray, D. J. Norris, and M. G. Bawendi, J. Am. Chem. Soc. 115, 8706 (1993).

${ }^{19}$ V. L. Colvin, A. N. Goldstein, and A. P. Alivisatos, J. Am. Chem. Soc. 114, 5221 (1992).

${ }^{20}$ W. W. Yu, L. Qu, W. Guo, and X. Peng, Chem. Mater. 15, 2854 (2003).

${ }^{21}$ Y. M. Niquet, C. Delerue, G. Allan, and M. Lannoo, Phys. Rev. B 65, 165334 (2002).

${ }^{22}$ M. Lannoo, C. Delerue, and G. Allan, Phys. Rev. Lett. 74, 3415 (1995).

${ }^{23}$ A. Franceschetti and A. Zunger, Phys. Rev. B 62, 2614 (2000).

${ }^{24}$ C. Delerue and M. Lannoo, Nanostructures, Theory and Modelling (Springer-Verlag, Berlin, 2004).

${ }^{25}$ Y. M. Niquet, Ph.D. thesis, l'Université des Sciences et Technologies de Lille, France, 2001.

${ }^{26}$ C. Wang, M. Shim, and P. Guyot-Sionnest, Science 291, 2390 (2001).

${ }^{27}$ D. Yu, C. Wang, and P. Guyot-Sionnest, Science 300, 1277 (2003).

${ }^{28}$ C. Wang, M. Shim, and P. Guyot-Sionnest, Appl. Phys. Lett. 80, 4 (2002).

${ }^{29}$ S. W. Wu, G. V. Nazin, X. Chen, X. H. Qiu, and W. Ho, Phys. Rev. Lett. 93, 236802 (2004). 